www.jmscr.igmpublication.org

Impact Factor 5.84

Index Copernicus Value: 83.27

ISSN (e)-2347-176x ISSN (p) 2455-0450

crossref DOI: _https://dx.doi.org/10.18535/jmscr/v5i3.166

Journal Of Medical Science And Clinical Research

\title{
Visual Outcome and Complications in Patients with Ocular Chemical Injuries
}

\author{
Authors \\ Dr Nimmy Merin Mathew, Dr Mallika. O.U \\ Department of Ophthalmology, Govt. Medical College, Thrissur \\ Corresponding Author \\ Dr Nimmy Merin Mathew \\ Puliyampallil House, Manjoor P.O, Kuruppanthara, Kottayam Dist, Kerala. PIN-686603 \\ Email: nimmimerin87@gmail.com, Phone-9446962227
}

\begin{abstract}
Purpose: To assess the visual outcome and the severity of injury and complications in patients with ocular chemical injury

Materials and Methods: In a tertiary hospital based prospective single cohort study all patients who presented with ocular chemical injuries to Ophthalmology outpatient department during a period of one and a half years were studied. Data regarding the history of injury with special emphasis to type of chemical, site and mode of exposure and time between injury and presentation to the hospital was taken. Visual acuity at the time of presentation was assessed and the severity of injury was studied by slit lamp examination. The patient was followed up on day1, day 3, at 2 weeks and at 2 months to assess the visual outcome and complications after the injury.

Results: among the 34 eyes with chemical injury, 52.9\% were alkali injuries,32.4\% were acid injuries and rest due to other chemicals. According to Roper Hall grading, grade 1 injury was present in $44.1 \%$ cases, grade 2 injury in $29.4 \%$ cases, grade 3 injury in $20.6 \%$ cases and grade 4 or severe injury was seen in only 2 eyes (5.9\%) cases. on review after 2 months, 30 eyes had best corrected visual acuity improved to 6/6 - 6/18 (88.2\%). Still, 3 eyes (8.8\%) had their visual acuity in the range of $6 / 24-3 / 60$ and one eye had visual acuity $<3 / 60$.

Conclusion: Alkali injuries were more and the incidence was more in the younger working population, with majority of injuries occurred in less than 40 years of age. The severity of injuries were less with most of them belonging to grade 1 and grade 2 injuries. Henceforth the visual outcome was better and complications were much less at 2 months follow up.
\end{abstract}

\section{INTRODUCTION}

The incidence of ocular chemical injuries is increasing nowadays due to rapid industrialization in developing countries. Various studies around the world have reported a wide range of results regarding the incidence of ocular chemical injury. Also this is one among the major causes for severe ocular morbidities including blindness particularly in the younger working population. ${ }^{1}$ Chemical injuries of the anterior segment of the eye are a common problem in the ophthalmology practice and can range from mild irritation to complete damage of the anterior segment. Delay in seeking proper management can result in severe vision 
threatening co-morbidities. It is well known that both strong acids and strong alkalies can cause severe eye injuries and, vision threatening complications can occur depending on the extent to which the eye surface is involved. The severity depends upon the nature, properties and concentration of the chemical, area of the affected surface, length of exposure and the presence of associated damage. ${ }^{2}$ The prognosis of chemical burns depends on the extent of the ocular surface damaged, the degree of intraocular penetration, and the concentration and nature of the agent involved. Chemical burns may be caused by either alkaline or acidic agents. The injuries caused by chemical burns to the eye can range from mild conjunctival or corneal epithelial damage to permanent blindness. Common alkaline agents include ammonium hydroxide used in fertilizer production, sodium hydroxide (caustic soda) used for cleaning drains and pipes, and calcium hydroxide found in lime, plaster and cement. Alkaline agents are particularly damaging as they have both hydrophilic and lipophilic properties, which allow them to rapidly penetrate cell membranes and enter the anterior chamber. Acids tend to cause less damage than alkalies as many corneal proteins bind acid and act as a chemical buffer. In addition, coagulated tissue acts as a barrier to further penetration of acid. Acid binds to collagen and causes fibril shrinkage. Considering their younger age, the long-term disabilities that follow ocular burns could dramatically affect the patients' lives. The goal of treatment is to minimize further damage to ocular surface and ultimately restore a normal ocular surface anatomy and visual function. Ocular chemical injuries are common in industries, construction workers, people working in laboratories, agriculture, fabric mills, automotive repair facilities, and cleaning and sanitizing crews. These can occur at varied circumstances as even at home. It is important that the availability of efficient precautionary measures can significantly diminish the incidence and severity of chemical injuries. Public awareness and legislation to use personal protective devices can reduce the occurrence of ocular injury. Chemical burns can produce devastating consequences for the ocular surface and periocular structures. Hence, adequate and timely treatment after an injury can reduce the severity of injury and development of serious complications. The overall goal of management should be restoration of the normal ocular surface anatomy and visual function. Ocular chemical injury can cause significant visual impairment in younger economically productive age group. Hence it is necessary to evaluate the damages caused by it and manage it with optimal intervention to restore vision to the maximum possible. Thus this study has been undertaken to know the patterns of ocular chemical injury, complications, their management and visual outcome.

Hypothesis: Severe ocular chemical injuries are associated with poor visual outcome.

\section{MATERIALS AND METHODS}

In a tertiary hospital based prospective single cohort study all patients who presented with ocular chemical injuries to Ophthalmology outpatient department during a period of one and a half years were studied. Patients with pre existing lenticular opacity, uveitis and glaucoma were excluded from the study. The estimated sample size is 30 patients, calculated using the formula $(4 p q) \div \mathrm{d} 2$, where $\mathrm{p}=$ incidence of complications in ocular chemical injury, $q=100-p, d$ $=$ clinically allowable error with a confidence interval of $95 \%$, power of study $90 \%$ and significance level of $5 \%$. This was based on the study done by Jiaxu Hong et al at Shanghai. ${ }^{3}$ Data regarding the history of injury with special emphasis to type of chemical, site and mode of exposure and time between injury and presentation to the hospital was taken. Visual acuity at the time of presentation was assessed and the severity of injury was studied by slit lamp examination. Roper Hall grading and Dua classification were used for assessing the extent of corneal injury and limbal ischemia. ${ }^{9,10}$. Patient 


\section{JMSCR Vol||05||Issue||03||Page 19379-19383||March}

was given treatment as needed based on the severity of injury and complications, if any. The patient was followed up on day 1 , day 3 , at 2 weeks and at 2 months to assess the visual outcome and complications after the injury. The obtained data was entered into the excel worksheet and analysis was done using SPSS software version 16 . The frequency and percentage of all relevant variables were calculated. Qualitative variables were assessed using Chi Square test. A p value less than 0.05 was considered as significant result

\section{RESULTS}

Figure 1 : Frequency of chemical injuries

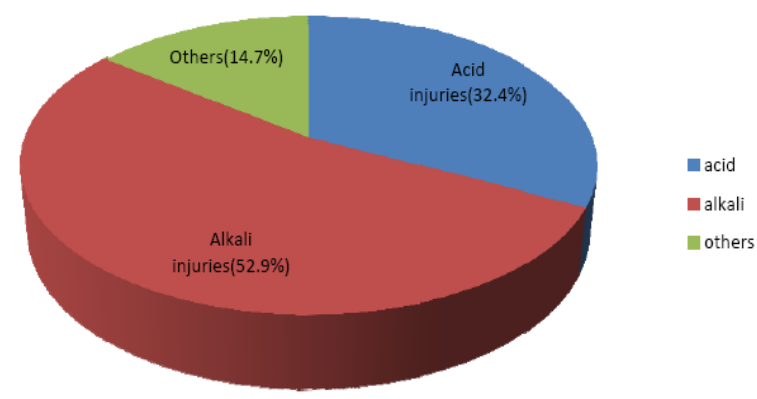

Figure 2. Occupation Vs Type of chemical.

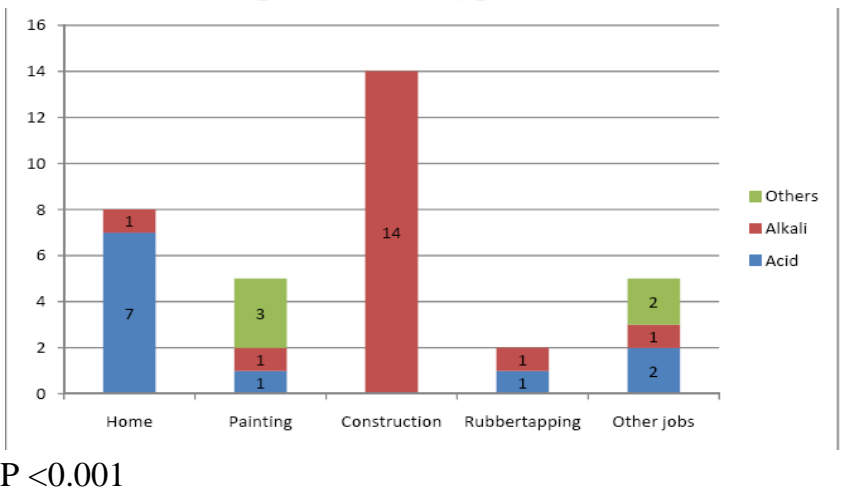

Figure 3. Roper Hall grading of ocular of chemical injuries.

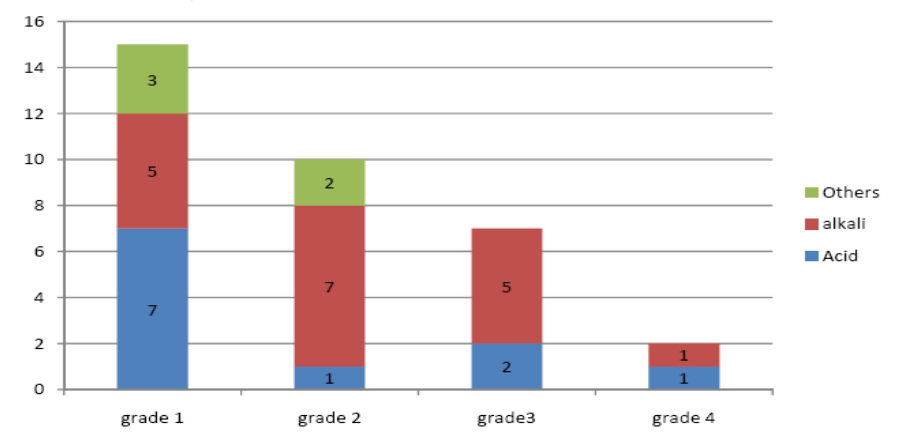

Figure 4: Time of initial presentation

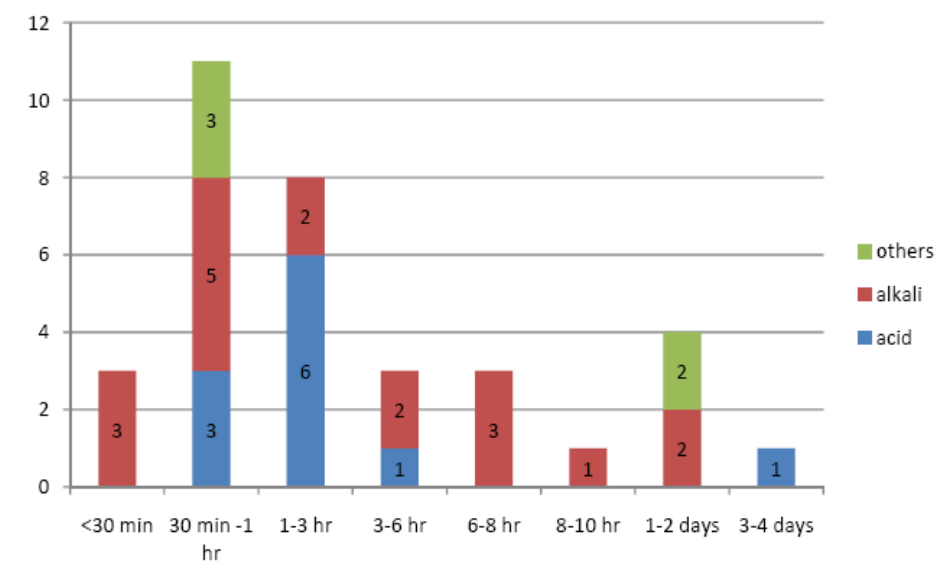

Figure 5: Best corrected visual acuity (BCVA) at 2 months

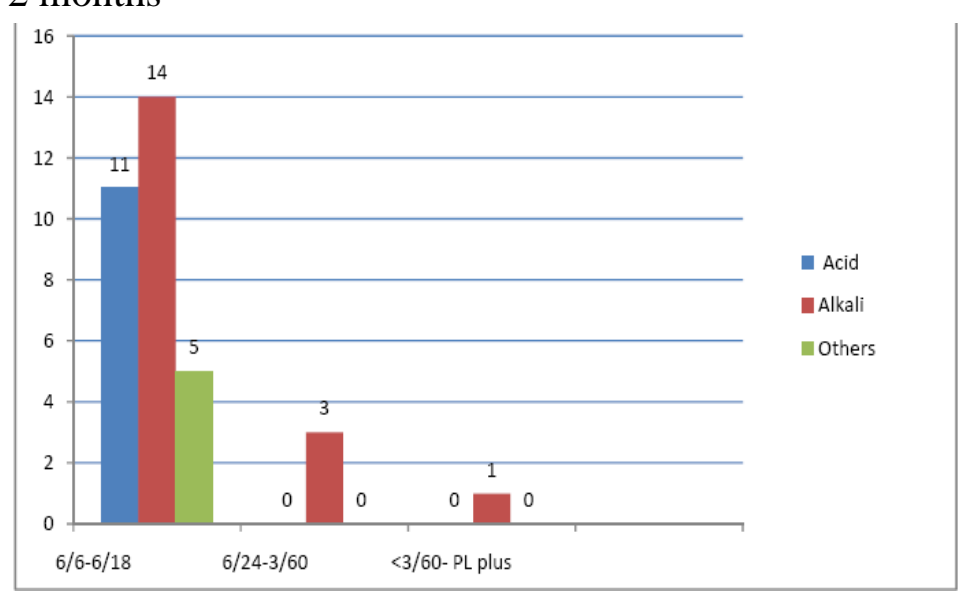

p- 0.600

\section{DISCUSSION}

In our study there were 26 males with chemical injury out of 30 patients and the incidence of the same was $76.4 \%$.In our study, among the 34 eyes with chemical injury, $52.9 \%$ were alkali injuries, $32.4 \%$ were acid injuries and rest due to other chemicals. In 1995 Kuckelkorn found that the majority of burns were caused by alkalis (62.4\%) and a smaller share by acids ( $21.8 \%$ ) and thermal agents (15. $8 \%$ ).4Majority of ocular chemical injuries in our study were in 11-30 years age group $(40 \%)$ and there were only one eye affected in the group of $<10 \mathrm{yrs}$ and $>70$ years. This was in agreement with the study of I M Shukla et al. ${ }^{5}$.In their study, they found that commonest age group for ocular injuries as third decade with an incidence of $29.5 \%$. In our study, among 34 eyes with chemical injury, 24 (70.6\%) had exposure to 
chemicals at work site and the rest occurred at home. Acid injuries were more common among the home injuries whereas alkali injuries predominated the work related injuries. This was in agreement with the study of Kuckelkorn et al and Hunt et al. ${ }^{4,6}$.In our study, according to Roper Hall grading, grade 1 injury was present in $44.1 \%$ cases, grade 2 injury in $29.4 \%$ cases, grade 3 injury in $20.6 \%$ cases and grade 4 or severe injury was seen in only 2 eyes (5.9\%) cases. This shows that majority of injuries were mild in our study. Dua classification also showed similar results in our study. In our study, on review after 2 months, 30 eyes had best corrected visual acuity improved to $6 / 6-6 / 18$ (88.2\%). Still, 3 eyes $(8.8 \%)$ had their visual acuity in the range of 6/24 - 3/60 and one eye had visual acuity $<3 / 60$. This was in agreement with the study of Subrata Das et al.7. At 3 months follow up, Subrata Das et al found that majority of patients had best corrected visual acuity in the range of 6/12 - 6/24 (56\%). Most of the injuries were minor and so $20 \%$ patients had visual acuity better than 6/9.In our study the main complications noted at 2 months follow up were symblepharon(5.9\%), cataract(5.9\%),

ectropion(2.9\%), corneal scar(26.5\%), dry eye $(26.5 \%)$ and secondary glaucoma $(2.9 \%)$. So the most common complications in our study were corneal scar and dry eye. This was in agreement with the result of Rasik B. Vajpayee et al and Subrata Das et al. ${ }^{7,8}$ The most common complications seen in the study of Rasik B. Vajpayee et al were central corneal opacification followed by symblepharon, entropion and elevated intraocular pressure.

\section{CONCLUSIONS}

In our study, alkali injuries were more and the incidence was more in the younger working population, with majority of injuries occurred in less than 40 years of age. Most of these injuries happened at construction work site and the result was statistically significant. But the severity of injuries were less with most of them belonging to grade 1 and grade 2 injuries. Henceforth the visual outcome was better and complications were much less at 2 months follow up. Considering the younger age group of patients affected, the injury can adversely affect their quality of life. Since majority of our population are literate and are living in a better socioeconomic conditions, they were aware of the consequences of ocular chemical injury and have seeked treatment as early as possible, reducing the severity of injury.

\section{REFERENCES}

1. Singh P, Tyagi M, Kumar Y, Gupta KK, Sharma PD. Ocular chemical injuries and their management. Oman J Ophthalmol. 2013;6(2):83-86.

2. Maskati BT, Adrianwala SD. Chemical burns of the conjunctiva and cornea. 1968;16(4):228-231

3. Hong J, Qiu T, Wei A, Sun X, Xu J. Clinical characteristics and visual outcome of severe ocular chemical injuries in Shanghai. Ophthalmology. 2010 Dec; 117(12):2268-72.

4. Kuckelkorn R, Kottek A, Schrage N, Reim M. Poor prognosis of severe chemical and thermal eye burns: the need for adequate emergency care and primary prevention. Int Arch Occup Environ Health. 1995;67(4):281-4.

5. Gomaa A, Comyn O, Liu C. Keratoprostheses in clinical practice - a review. Clin Experiment Ophthalmol. 2010 Mar;38(2):211-24.

6. Hunt JP, Calvert CT, Peck MD, Meyer AA. Occupation-related burn injuries. J Burn Care Rehabil. 2000 JulAug;21(4):327-32.

7. Das S, Kabir MF, Das J, Hannan AA, Chowdhury MW, Anwar SMN et al. Pattern of Chemical Ocular Injury: A Clinical Study. Chattagram Maa-O-Shishu Hospital Medical College Journal. 2014 Jan;13(1):42-45.

8. Vajpayee RB, Shekhar H, Sharma N, Jhanji V. Demographic and clinical profile 
of ocular chemical injuries in the pediatric age group. Ophthalmology. 2014 Jan;121 (1):377-80.

9. Dua HS, King AJ, Joseph A. A new classification of ocular surface burns. Br J Ophthalmol. 2001 Nov;85(11):1379-83

10. Roper-Hall MJ. Thermal and chemical burns. Trans Ophthalmol Soc U K. 1965;85:631-53. 